\title{
Facial Cellulitis and Thrombosis With Pulmonary Septic Emboli Mimicking Lemierre's Syndrome
}

\author{
Sabrina Arshed ${ }^{\mathrm{a}, \mathrm{b}}$, Mohammad Amir Hossain ${ }^{\mathrm{a}}$, Soaud Enakuaa ${ }^{\mathrm{a}}$, Sunil Tulpulea ${ }^{\mathrm{a}}$, Qiang Nai ${ }^{\mathrm{a}}$, \\ Mohammed Osman ${ }^{\mathrm{a}}$, Rafay Khan ${ }^{\mathrm{a}}$, Nazar Raoof ${ }^{\mathrm{a}}$, John Middleton ${ }^{\mathrm{a}}$, Purendu Sen ${ }^{\mathrm{a}}$, \\ Abdalla Yousif ${ }^{\mathrm{a}}$
}

\begin{abstract}
Lemierre's syndrome is characterized by thrombophlebitis of the internal jugular vein and bacteremia, typically caused by anerobic microorganisms following a recent pharyngeal infection, first described by Andre Lemierre as a clinical finding of "anerobic post-anginal sepsis" in 1936. Diagnosis is made by identifying thrombosis of the internal jugular vein; although variants of the disease with facial vein thrombosis have been reported, in addition to positive anerobic blood cultures, the most common causative organism remains Fusobacterium necrophorum. The mainstay of treatment remains prolonged antibiotic therapy.
\end{abstract}

Keywords: Lemierre's syndrome; Facial vein thrombus; Septic emboli; Atypical presentation

\section{Introduction}

Lemierre's syndrome is a condition characterized by clinical or radiological evidence of thrombosis of the internal jugular vein and bacteremia primarily caused by anerobic organisms following a recent oropharyngeal infection. Throughout the 1980s - 1990s, the disease became known as forgotten disease, as it was very rare; however, in the last 15 years, the frequency of Lemierre's syndrome has increased, possibly due to antibiotic resistance.

Variants of Lemierre's disease showing facial vein thrombosis have been reported in multiple case reports, and by Andre Lemierre himself. These variants, however, typically present with a similar prodrome of pharyngitis prior to thrombosis formation. We present a case of a patient who presented with septic thrombosis of the facial vein, beginning shortly after picking an infected pimple within the nares, associated with

Manuscript accepted for publication January 19, 2016

araritan Bay Medical Center, Perth Amboy, NJ, USA

${ }^{b}$ Corresponding Author: Sabrina Arshed, Internal Medicine Residency Department, Raritan Bay Medical Center, 530 New Brunswick Avenue, Perth Amboy, NJ 08861, USA. Email: Sabrina.arshed@gmail.com

doi: http://dx.doi.org/10.14740/jmc2422w metastatic septic emboli without a prodrome of pharyngeal infection, representing the first documented case of a disease process mimicking Lemierre's syndrome.

\section{Case Report}

We present a case of a previously healthy 29 -year-old male, admitted for progressively worsening swelling over the left side of the face that began 5 days prior to admission. The patient noted that approximately 1 week prior to admission, he had picked a pimple on the lateral wall within the left nares. Three days prior to admission, he was diagnosed with facial cellulitis and discharged from the ED with oral amoxicillin.

On admission, and throughout the hospital course, the patient's vital signs remained stable, and white blood cell count peaked at 11.1. However, erythrocyte sedimentation rate (ESR) was $60 \mathrm{~mm} / \mathrm{h}$ and C-reactive protein was $3.4 \mathrm{mg} / \mathrm{dL}$.

The patient was started on intravenous clindamycin and vancomycin. A computed tomography (CT) scan with contrast of the facial bones was done showing left facial preseptal soft tissue swelling with a thrombosis of the second-order branch of the left facial vein (Fig. 1), and mild left-sided adenopathy. Coagulopathy and vasculitis workup was done, and found to be within normal limits.

Furthermore, the patient's alkaline phosphatase was elevated on admission. Hepatitis panel was negative, right upper quadrant ultrasound was normal, and contrast-enhanced abdominal CT scan showed multiple bilateral lung base nodules, one of which had a central cavitation. A dedicated CT scan of the chest was done revealing numerous $1-3 \mathrm{~cm}$ peripheral predominant pulmonary nodules, some of which were cavitary, with an appearance most characteristic of septic emboli (Fig. 2). Chest $X$-ray done 3 days prior to admission was normal, without any evidence of any cavitary lesions.

The patient had emigrated from Mexico 8 years prior to admission, and had not had any recent travel or exposure to tuberculosis; nevertheless, a PPD was placed and found to be negative. Quantiferon was negative and acid fast sputum smear and cultures were also negative, effectively ruling out tuberculosis. Serum ACE was normal, echinococcus and cysticercosis serology was negative and rapid HIV was also found to be negative. Blood cultures drawn on admission, after receiving intravenous antibiotics, were found to be negative. 


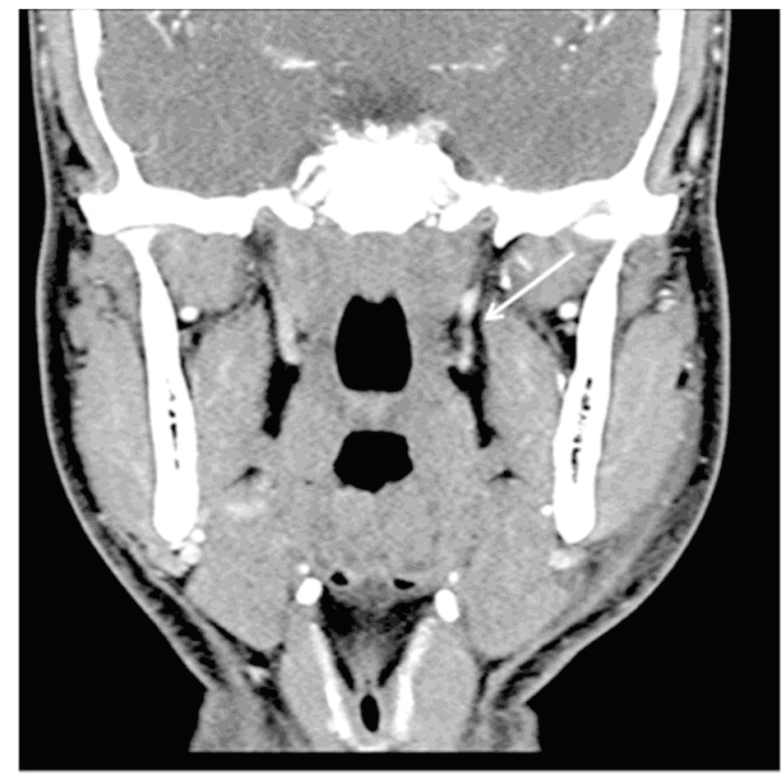

Figure 1. CT scan of the facial bones demonstrating a left facial/preseptal soft tissue swelling, with an associated thrombosis of a secondorder branch of the left facial vein.

Sputum culture was negative. Anerobic culture from the left nares grew Propionibacterium acnes.

After nearly 1 week of intravenous antibiotics, the patient's facial swelling significantly improved. The patient was diagnosed with a Lemierre-like syndrome and was discharged on oral clindamycin for 2 weeks, and would be followed up as an outpatient. One month after discharge, a repeat CT scan of the chest was done showing an overall decrease in the size of the multiple pulmonary nodules (Fig. 3).

\section{Discussion}

Lemierre's syndrome, once called the forgotten disease, is considered rare in our current antibiotic era, but more and more cases are being seen, likely due to increased antibiotic resistance [1]. Lemierre's syndrome is a condition typically characterized by septic thrombosis of the internal jugular vein and bacteremia caused primarily by anerobic organisms, the most common being Fusobacterium necrophorum (FN), following an acute pharyngeal infection. Lemierre's syndrome may lead to metastatic abscesses and distant septic emboli $[2,3]$.

Lemierre's syndrome usually presents with persistent fever, followed by acute pharyngitis and eventual sepsis. After this, neck swelling and tenderness tends to develop and the use of ultrasonography or CT scan with contrast can confirm internal jugular vein thrombosis. Confirmation of Lemierre's syndrome is made with positive blood cultures growing FN [2].

In a review of more than 100 cases, it was found that the Lemierre's syndrome most commonly presents during the second decade of life, in approximately $51 \%$ of cases [3]. Fusobacterium species, more specifically FN, which is normal flora

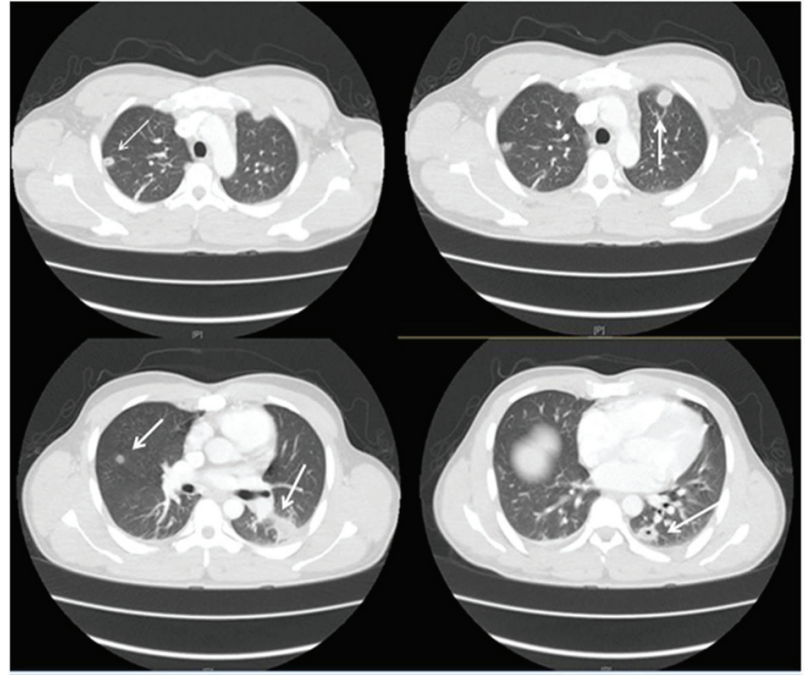

Figure 2. CT scan of the chest with contrast demonstrating numerous $1-3 \mathrm{~cm}$ peripheral predominant pulmonary nodules, some of which are cavitary, most characteristic of septic emboli.

of the oropharynx, is responsible for the majority of reported cases; however, up to $30 \%$ of patients will have polymicrobial bacteremia with both gram positive and gram negative anerobic organisms [3].

Metastatic septic emboli have been seen in approximately $63-100 \%$ of patients, the lungs being the most common site, followed by major joints [3]. Septic metastatic infections have also been described in the liver, pericardium and brain, but are much rarer [3].

Treatment of Lemierre's disease remains prolonged therapy with antibiotics directed at anerobes, including clindamycin, metronidazole, imipenem or cefodizime [2]. The use of anticoagulation in Lemierre's syndrome remains controversial due to the lack of clinical evidence and lack of any controlled studies [3, 4]. Surgical therapy may have a role in the drainage of abscesses in the neck, and previously ligation of the internal jugular vein is the mainstay of treatment; however, ligation is now reserved for patients with persistent emboli, despite antibiotic treatment $[3,5]$.

There have been case reports of atypical variants of Lemierre's syndrome with isolated facial vein thrombophlebitis. In these cases, the patients present with the typical prodrome of Lemierre's syndrome; however, the thrombophlebitis occurs in a less common location, the facial vein. Andre Lemierre had previously described isolated facial vein thrombosis, and other than that it has only been rarely mentioned in literature [6].

However, in our case, our patient had negative blood cultures, which was to be expected as the patient was treated with oral antibiotics 3 days prior to admission and was given his first dose of intravenous antibiotics prior to obtaining blood culture samples. Furthermore, our patient's source of infection was an infected pimple within his nose that he picked, leading to a septic thrombus within the facial vein, and at no point had any symptomology of pharyngitis. Nasal cultures also showed the growth of Propionibacterium acnes, which 


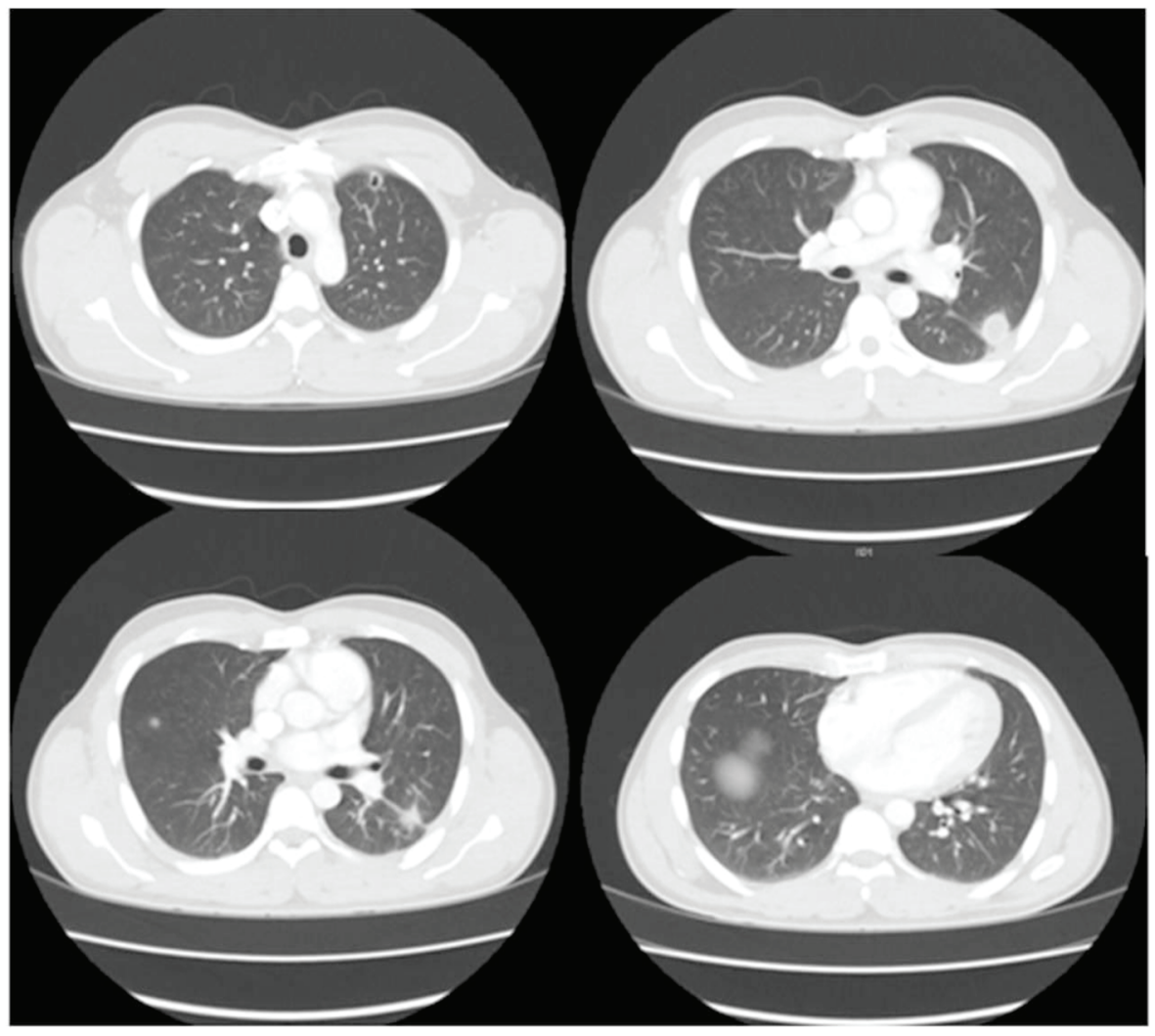

Figure 3. CT scan of the chest with contrast demonstrating overall decrease in size of the multiple pulmonary nodules, some of which have progressed into purely cavitary lesions slightly thick walled, while others remain solid, but smaller.

when the pimple was ruptured, may have invaded the blood stream via nasal capillaries, subsequently leading to a left facial vein thrombosis, leading to metastatic septic emboli to the lungs. Given our patient's presentation and course, we defined our patient's condition as a Lemierre-like syndrome, given the presence of septic thrombosis of the facial vein and metastatic septic emboli.

\section{Conclusion}

Lemierre's syndrome presents mostly in young healthy individuals with a history of recent oropharyngeal infection, with evidence of internal jugular vein thrombosis and anerobic bacteremia. However, case reports have proven that the facial vein may also be affected in variants of Lemierre syndrome. Our case represents a condition with findings similar to that of Lemierre's syndrome with facial vein thrombophlebitis and septic emboli without any prodrome of recent infection. Given this presentation, it is important for clinicians to keep in mind Lemierre-like syndromes may occur in patients without any recent pharyngitis, and early detection and proper management may significantly decrease mortality and morbidity in this patient population.

\section{Conflicts of Interest}

The authors of this paper declare no conflict of interest.

\section{References}

1. Karkos PD, Asrani S, Karkos CD, Leong SC, Theochari EG, Alexopoulou TD, Assimakopoulos AD. Lemierre's syndrome: A systematic review. Laryngoscope. 2009;119(8):1552-1559.

2. Singhal A, Kerstein MD. Lemierre's syndrome. South Med J. 2001;94(9):886-887.

3. Eilbert W, Singla N. Lemierre's syndrome. Int J Emerg Med. 2013;6(1):40.

4. Kisser U, Gurkov R, Flatz W, Berghaus A, Reichel O. Lemierre syndrome: a case report. Am J Otolaryngol. 2012;33(1):159-162.

5. Lemierre A. On certain Septicemias due to Anaerobic Organisms. Lancet. 1936;227:701-703.

6. Iizuka T, Nagaya K, Sasaki D, Haruyama T, Kojima M, Isogai $\mathrm{H}$, Yoshikawa $\mathrm{H}$, et al. Atypical Lemierre syndrome, thrombophlebitis of the facial vein. Am J Emerg Med. 2013;31(2):460 e461-463. 